\title{
VALORACIÓN ECONÓMICA DEL DELITO DEL HOMICIDIO EN CARTAGENA ${ }^{1} .1995$ - 2005
}

\author{
FREDI GOYENECHE GONZÁLEZ* \\ JERRY PARDO GÓMEZ \\ OSWALDO MÁRMOL PÉREZ ${ }^{* * * *}$
}

Recibido 9 de Octubre de 2010/Enviado para Modificación 19 de Noviembre de 2010/Aceptado 3 de Diciembre de 2010

\begin{abstract}
RESUIMEN
En el presente documento, se analizan los costos económicos de los homicidios en el distrito de Cartagena para el periodo 1995 - 2005, los cuales se determinan con el cálculo de los Años Potenciales de Vida Perdidos (APVP), a partir de las bases de datos de homicidios del periodo 1995 - 2005 procesadas por el COSED las cuales permiten caracterizar la violencia homicida a partir de un conjunto de variables como hora de ocurrencia de los hechos, contextos, barrios de ocurrencia y clase de lugar, entre otras. Dentro de los principales resultados se comprobó que entre 1995 y el 2005 se observó un promedio anual de 8.452 APVP, siendo 11.018 el valor máximo alcanzado en el año 2003, y 7.010 el valor mínimo el cual se obtuvo en el año 1998.
\end{abstract}

Palabras Clave: Costos económicos, años potenciales de vida perdidos, homicidios.

Clasificación JEL: A1, B41, O15

1 El presente artículo es producto de una investigación mayor realizada en el año 2006, en
donde no sólo se estimó la valoración económica de los homicidios en la ciudad sino que
además se cálculo su incidencia sobre la inversión. Este artículo fue construido por los autores
en el marco del Grupo de Investigación Economía, Violencia y Ciudad con el apoyo muy es-
pecial de los Estudiantes de último semestre del Programa de Economía de la Universidad de
Cartagena, Jorge Leonardo Castillo Loaiza y Andrés Mauricio Castaño Zuluaga.
*Facultad de Ciencias Económicas - Universidad de Cartagena. Correo Electrónico: fredigoy-
eneche@gmail.com.
** Departamento de Investigaciones Económicas y Sociales - DIES. Universidad de Cartagena.
Correo Electrónico: jerry_pardo@diesudc.com
*** Consultor Independiente, Asesorías M\&P. Correo Electrónico: oswmarmol@hotmail.com. 


\begin{abstract}
This paper analyzes the economic costs of the homicides in the District of Cartagena for the period 1995 - 2005, which are determined by the calculation of Years of Potential Life Lost (YPLL) from the database of homicides the period 1995 - 2005 processed by the COSED, which can characterize the homicidal violence from a set of variables such as time of occurrence of the events, contexts, neighborhoods of occurrence and type of place, among others. Among the main results found that between 1995 and 2005 had an average annual YPLL 8.452, being 11.018 the highest level reached in 2003 and 7.010 the minimum value which was obtained in 1998.
\end{abstract}

Key Words: Economic costs, years of potential life lost, murders

JEL Classification: A1, B41, O15

\title{
INTRODUCCIÓN
}

El contexto de desarrollo de largo plazo económico y social en Colombia, ha estado signado por elementos seminales que tienen que ver con la violencia. El mismo proceso originario de lo que luego sería la territorialidad de la República de Colombia se define a partir de las connotaciones de la conquista, colonización y el surgimiento de una nueva sociedad como la nuestra y como la del conjunto de países de América Latina: Desplazamiento y Violencia. Esta violencia está básicamente fundada en el desconocimiento que tiene el derecho a la vida, a la propiedad y a la honra. En este contexto, la figura del homicidio aparece determinada por normas superiores que lo definen de tal manera que estos, a su vez, se encuentran en el punto de partida de una problemática que no será nunca suficientemente estudiada.

En el presente estudio, se aplican una serie de cálculos con la finalidad de estimar el valor monetario de la vida de una persona que ha sido víctima de un homicidio, o dicho de otra manera, se plantea una forma de calcular el costo económico de las muertes por homicidios en la ciudad de Cartagena durante el periodo 1995 - 2005, bajo la metodología (APVP)². Conjuntamente, se plantea como hipótesis central el hecho de que el costo económico que constituye para la sociedad una muerte por homicidio está representa-

\footnotetext{
2 Años Potenciales de Vida Perdidos (APVP), denominación utilizada por La Organización Panamericana de la Salud en estudios de epidemiología bajo el software EPIDAT 3.1
} 
do por la contribución que esa persona pudo haber aportado en vida de no haber sido asesinada, o sea la cantidad de APVP.

Dentro de la literatura universal, desarrollada hasta ahora para aproximarse al costo económico que representa para la sociedad una muerte por homicidio, la teoría de los APVP propuesta por primera vez en 1947, utilizada en España y en otro países de Europa y que en Suramérica, ha sido ampliamente utilizada por el Ministerio de Salud de Chile (MINSAL) en estudios que se remontan al año 1996, es tal vez la más utilizada y con la que se han obtenido resultados mucho más certeros relacionados con esta temática. Un APVP es un año de vida saludable perdido por morir prematuramente" (1), o como optaremos por llamarlo en este documento un "Año Potencial de Vida Perdido" y su cálculo nos permite conocer el impacto que tienen diferentes tipos de muertes dentro del contexto social de una comunidad en específico.

Además de todo lo anterior, en el presente documento se presenta un análisis que caracteriza el perfil de los homicidios en Cartagena, basado en las cifras publicadas por el Centro de Observación y Seguimiento del Delito, COSED; En la parte metodológica se explica la forma en la que se puede obtener una aproximación del costo económico de los homicidios. Al final se plantean unas conclusiones y recomendaciones que podrían servir de base de análisis en estudios posteriores.

\section{REVISIÓN DE LA LITERATURA}

Pocos son los estudios, que hasta ahora utilizan la teoría económica como herramienta para explicar el fenómeno del crimen y la violencia en Colombia y mucho menos en Cartagena, e incluso los que se han realizado solo se centran en explicar las causas de la violencia generadas básicamente por el narcotráfico y la subversión y algunos otros fenómenos de carácter social y psicológicos. A continuación se hace una breve descripción general de algunos de los textos más importantes desarrollados hasta el momento acerca de la temática de los homicidios, sus costos, implicancias, aspectos sobresalientes y más.

Carlos Mario Gómez, en un ensayo titulado ECONOMÍA Y VIOLENCIA en Colombia y publicado en el libro ECONOMÍA CRIMEN Y CONFLICTO editado por Astrid Martínez Ortiz (2005) (2), desarrolla el estudio del fenómeno de la violencia, utilizando la teoría económica como herramienta de análisis, al tratar de comparar la violencia con la desigualdad y afirmar que la hipótesis de que la desigualdad es causa de la violencia (Hipótesis de privación relativa) y que a pesar de que existe una elevada correlación entre 
estas dos variables, no es suficiente razón científica para afirmar que la primera sea consecuencia de la segunda. Básicamente esto se explica porque los niveles de desigualdad en la distribución del ingreso de Colombia con relación a los de otros países de Latinoamérica donde no hay conflicto armado, o ya se han superado, no son muy diferentes. De esta manera sugiere como igual de plausible la hipótesis contraria que la violencia es causa de la desigualdad. En este sentido el consenso acerca de la validez o no de la hipótesis de la privación relativa ha conducido a una hipótesis alternativa enmarcada en lo que se conoce como la "escuela de movilización de recursos".

Por otro lado, este mismo autor cuando se refiere a la violencia y la economía y en búsqueda de explicaciones al fenómeno de la violencia admite los aportes importantes del análisis económico en este campo de estudio. Partiendo de la denominada Economía del crimen cuyo principal representante es Becker (1968) (3) y su principal sustento es la denominada "hipótesis de disuasión", en la que se considera que el delincuente realizará sus delitos siempre y cuando después de un análisis costo beneficio encuentre que el costo de cometer el crimen, es decir la probabilidad de ser capturado, sea menor que los beneficios obtenidos.

En este sentido, son las autoridades judiciales las encargadas de hacer que la probabilidad de que los criminales sean capturados aumente para lograr que la criminalidad disminuya y esto implica ajustes en el gasto público. Luego entonces, el Estado no permitirá que el fenómeno del crimen sea permanente que es lo que la economía del crimen pretende explicar.

En el ámbito local se destaca un artículo, escrito por Augusto Otero y Aarón Espinosa denominado APUNTES PARA ENTENDER LA VIOLENCIA Y LA CRIMINALIDAD EN CARTAGENA (2001), en donde los autores realizan un análisis descriptivo sobre la problemática de la violencia en Cartagena partiendo de un estudio realizado por un importante gremio de la ciudad que arroja resultados alarmantes sobre todo para el sector turístico que temía, que por esto, el sector terminara siendo afectado como lo fue por los carros bomba puestos en el sector histórico de la ciudad. Hay que hacer notar algunos aspectos importantes con relación a este documento: 1) En el desarrollo del análisis utilizan cifras de Medicina Legal para calcular la tasa de homicidios, las cuales no están del todo depuradas y arrojan unos valores para las tasas que sobreestima el valor real de las mismas. Así, al hacer comparaciones con las tasas de homicidio de las principales ciudades de Colombia, Latinoamérica y el mundo, el análisis termina apartándose un poco de la realidad. 
Entre los aportes más significativos del documento, se destaca el tratar de caracterizar la naturaleza de la violencia homicida en Cartagena, encontrando que desde el año 2000 hasta el año 2002 el ajuste de cuentas es el principal contexto en que se realizan los homicidios representando este móvil el 55.7\% del total de los homicidios del año 2000, el 34.7\% del año 2001 y el $44.2 \%$ del año 2002, seguido por las riñas con el 13\% de los homicidios en el 2000, el $25.7 \%$ de los homicidios en el 2001 y el 13.3\% de los homicidios en el 2002, y a partir de allí sugiere las siguientes hipótesis ${ }^{3}$ :

$\checkmark$ "La violencia homicida en Cartagena, se caracteriza por la poca confianza de los individuos en su sistema judicial y por ello hay un elevado número de homicidios por ajuste de cuentas y la consecuencia más importante de esto es que como no existen mecanismos efectivos de disuasión, el homicida actúa como si le fuera rentable asesinar ya que la probabilidad de ser capturado es mínima".

$\checkmark$ "Las riñas aunque en menor escala como propulsora de homicidios abre la discusión sobre si se utiliza una de las teoría más populares de la economía del crimen la teoría de las "causas objetivas". Según esa teoría la violencia es un fenómeno cotidiano y generalizado, resultado de la intolerancia, la delincuencia común y el consumo de alcohol".

$\checkmark$ "Los homicidios por terrorismo están asociados a la mayor penetración del conflicto armado en las ciudades".

Otro estudio realizado en el ámbito local, se tituló FUNDAMENTOS ECONÓMICOS DE LA CRIMINALIDAD EN CARTAGENA DE INDIAS, por Antonella Farha Louis de la Universidad Tecnológica De Bolívar, 1997 (4). Este trabajo relaciona el crimen con el crecimiento económico fundamentándose en las teorías del desarrollo económico y en la economía del crimen de Gary Becker (3). Este trabajo no relaciona el crimen con variables económicas como la inversión, ni con variables sociales como la educación la pobreza y el desplazamiento.

Por último, en lo que concierne a la recolección análisis e interpretación de las estadísticas de muertes por causas externas donde se incluyen los homicidios se destaca el trabajo realizado por el equipo del CENTRO DE OBSERVACIÓN Y SEGUIMIENTO DEL DELITO, COSED, y los comentarios y análisis realizados por el profesor Fredi Eduardo Goyeneche González (5) en los cuales plantea posibles relaciones de causalidad entre la pobreza y la violencia en la ciudad y recomienda políticas públicas tendientes a enfrentar

3 El lector debe recordar que las cifras utilizadas en este documento se encuentran un poco desfasadas con relación a las cifras oficiales publicadas por el COSED, aunque en este caso la caracterización coincide en gran parte. 
el fenómeno de la criminalidad y la violencia, teniendo en cuenta la "multidimensionalidad" de causas y consecuencias de ella (6). Con ello recomienda a su vez una orientación del Gasto público en seguridad de manera integral, que pueda ser capaz de atender a todos y cada uno de los demandantes de seguridad en el distrito sin prescindir de género, raza o nivel económico.

En los informes del COSED, se analizan mensualmente las cifras sobre el comportamiento de los homicidios en el distrito, haciéndole seguimiento a una serie de variables como la edad y sexo de las víctimas, el barrio de ocurrencia de los hechos, el tipo de arma utilizada, el contexto, entre otras.

En cuanto a la determinación de los costos económicos de los homicidios, encontramos como antecedente un importante estudio realizados en el ámbito internacional, por Mauricio Olavarría Gambi con el apoyo del ILPES, titulado COSTOS ECONÓMICOS DE LOS DELITOS EN CHILE 2005 (7).

Este estudio, se aborda la problemática de los delitos desde la perspectiva económica haciéndose el siguiente interrogante "¿Cuál ha sido el costo económico de la actividad criminal en Chile en los años 2000, 2001 y 2002?". Estos delitos primero los clasifica como 1.) Los costos anticipados del delito: Que se refiere a los gastos que hacen los ciudadanos y las instituciones para evitar ser víctimas del delito. 2) Costos a consecuencia del delito: Que se refiere a todos los costos directos que produce la acción delictiva incluida la pérdida de la vida, y aquí entran los homicidios. 3) Costos en respuesta al delito: Consiste en los gastos que se incurre para perseguir la responsabilidad de los delincuentes. Por otro lado, para el caso de los homicidios se decide por utilizar la metodología del AVPM, que representa los años de vida perdidos por muerte prematura, considerando la esperanza de vida observada y la tabla de mortalidad ajustada por estructura de edad como una proxy de la fecha de muerte, y además sostiene que la pérdida económica está determinada por el ingreso no percibido en los AVPM, tomando el ingreso per cápita como proxy para estimar el ingreso no percibido. Esta metodología hace parte de un concepto mayor la AVISA (años de vida saludables perdidos).

En otro estudio, Iván Silva Lira, director de Desarrollo y Gestión local, ILPES, prepara un documento titulado COSTOS ECONÓMICOS DE LOS DELITOS, NIVELES DE VIGILANCIA Y POLÍTICAS DE SEGURIDAD CIUDADANA EN LAS COMUNAS DEL GRAN SANTIAGO (8). Empieza preguntándose cuál es el monto de los ahorros que se podrían generar en términos de pérdidas materiales, perdidas de vida, atención a heridos y tiempo de trabajo etc. versus el gastos de inversión que habría que hacer para que los mencionados ahorros se produjeran. La respuesta la halla afir- 
mando que se trata de un análisis de costo beneficio que puede resultar en inversiones con una alta tasa de rentabilidad social.

A partir de esta tentativa, construye una metodología para calcular los costos económicos de los delitos que incluye los costos de los accidentes y los costos contra la persona y la propiedad (solo reseñaremos estos últimos y en especifico los costos de los homicidios por ser el objetivo de nuestra investigación) para cada una de las comunas del gran Santiago.

La metodología del cálculo de los homicidios la asemeja a la pérdida de la vida por accidente y define el costo económico de este delito como "al menos el valor anticipado de sus ingresos futuros" (8). Bajo esta definición supone que la sociedad asigna un valor igual a todas las personas independientemente de su edad y considera como base para el cálculo de los ingresos futuros una vida útil promedio de las personas de 30 años. De acuerdo a la teoría económica del costo de oportunidad del tiempo en esta metodología se le da al ocio el mismo valor monetario que al tiempo trabajado. Entonces el costo de la muerte anticipada se calcula como el doble del valor actualizado de los ingresos futuros.

\section{METODOLOGIA PARA EFECTOS DE LOS CÁLCULOS DEL INDICADOR APVP}

El indicador APVP, que referencia los Años Potenciales de Vida Perdidos, es calculado con referencia a una población sobre la cual se desea obtener el número total de años perdidos por cada una de las muertes ocurridas por una causa determinada. Para efectos económicos el APVP se utiliza para acercarse al costo en términos de pérdida de bienestar, es decir, el costo para la sociedad que se deriva de una muerte por una causa determinada a partir de los años que no pudo vivir debido a la causa de muerte y los ingresos que no pudo percibir durante esos años.

Para el cálculo de los APVP en este documento, utilizaremos como límite inferior 1 año y como límite superior los 70 años, además de la tabla de mortalidad ajustada por estructura de edad para poder efectuar los cálculos de la tasa de APVP ajustada, con la finalidad de hacer comparaciones entre poblaciones con estructuras diferentes. Esta tabla es la de "Población Estándar Mundial de Waterhouse", utilizada por la OMS y OPS. Dado que la pérdida económica está determinada por los ingresos no percibidos durante los APVP, comúnmente se utilizarán como proxy de los ingresos no percibidos el PIB per cápita o el salario mínimo vigente. 
El APVP también puede ser calculado por géneros con la finalidad de saber qué grupo poblacional, según sexo, está siendo mayormente afectado por la causa de muerte determinada. "Los APVP tienen la ventaja de ser de fácil cálculo ya que requieren solamente de las defunciones por edad y de la población total. Sin embargo, como en cualquier estudio basado en datos de salud, la calidad del indicador dependerá de la calidad y cobertura de los datos que lo componen" (9).

Para el cálculo se procede de la siguiente forma:

$$
A P V P=\sum_{i=I}^{L}[(L-i) \times d]
$$

Donde:

$$
\begin{gathered}
\mathrm{i}=\text { es la edad límite inferior establecida } \\
\mathrm{L}=\text { es la edad límite superior establecida } \\
\mathrm{I}=\text { es la edad de la muerte } \\
\mathrm{d}=\text { es el número de defunciones a la edad } \mathrm{i}
\end{gathered}
$$

Los datos pertinentes para el cálculo del APVP, como las edades por rangos de las víctimas de los homicidios se obtendrán de la base de datos del COSED, el PIB per cápita nacional de las estadísticas del banco de la república y los datos poblacionales y de inversión de las bases de datos de la Secretaria de Planeación Distrital, DADIS y la Cámara de Comercio de Cartagena. El procesamiento de los datos para el cálculo del indicado APVP se hará a través del Software EPIDAT 3.1 y GESMOR que ofrecen la opción para el cálculo de dicho indicador.

\section{HOMICIDIOS EN CARTAGENA DE INDIAS: CIFRAS Y ANÁLISIS PERIODO 1995 - 2005}

Los resultados obtenidos muestran que en Cartagena de Indias durante 1995 - 2005, ocurrió un total de 2429 muertes por homicidios, de las cuales 226 ocurrieron durante el último año y 248 casos en el primero, 1995 (Ver Anexo No. 1). Se observa además que históricamente el mes de diciembre ha sido el de mayor ocurrencia de casos de homicidios, reportando 249 casos de los 2429 y representando el $10.25 \%$ del total. Esta incidencia de casos podría ser explicada, entre muchas razones, por la gran demanda de personas y afluencia de turismo a fin de año que se manifiesta de forma progresiva en la ciudad. Para explicar esta aseveración nos basamos en las 
cifras de entrada de vehículos que nos suministra la policía de carreteras en las que se observa que durante el 2003 y el 2005 entró a la ciudad un total de 168.440 vehículos en los meses de Diciembre (13.755 vehículos más que los reportados en los meses de Noviembre), pasando de 45.490 en el primer año a 72.493 vehículos en el segundo año (10).

A su vez, el año 2003 se constituye en el año de mayor ocurrencia de casos de homicidios con un total de 293 muertes, de las cuales 37 ocurrieron durante el mes de enero que a su vez representó el mes de mayor ocurrencia de casos en ese año. Contradictorio a la situación local, el 2003 para Colombia significó el año de menor tasa de homicidios de los últimos 18 años, tal y como lo revelan las cifras de la Policía Nacional, explicándose ello por el aumento considerable que tuvo el pie de fuerza y la cobertura en el país, dada la política de seguridad implementada por el presidente (12). En este sentido y teniendo en cuenta la óptica de la teoría de Becker, la probabilidad de captura para el delincuente en este periodo aumentó, ya que las cifras muestran que gracias a la política de aumento en la seguridad, durante el 2003 se logró un total de 1.810 capturas más que durante el 2002, la confianza de la población civil en las fuerzas militares obtuvo 80 puntos positivos, el pie de fuerza nacional se aumentó en un 17\% (56.000 nuevos uniformados), se consigue la aprobación de la Ley de Extinción de Dominio y se aumenta la pena máxima a 60 años.

Muy contrario a ello, ninguna de estas medidas tuvo impacto alguno sobre la incidencia de homicidios en Cartagena, en tanto que la teoría de Becker no ayuda mucho a explicar el porqué del comportamiento de las tasas de homicidios a nivel local, aunque sí para el caso nacional. La verdadera explicación o causa de estas fluctuaciones, para el caso de Cartagena, puede hallarse al interior de una posible relación de causalidad entre los niveles de pobreza y la violencia, lo que concuerda más con la teoría de Broken Windows de Wilson y Kelling, (1982) (12-13) que analizaremos más adelante.

De igual forma, la gráfica que se muestra a continuación (gráfica No.1) muestra la evolución anual de las muertes por homicidios desde 1995 hasta el 2005 y de igual forma muestra el comportamiento de la tasa de homicidios para ese periodo. Se observa que la cifra interanual se ubicó entre los 180 (en 1998) y 293 casos (en 2003), con diversas fluctuaciones en cada uno de los años y pasando de 248 casos en el 1995 a 226 casos en el 2005, lo que representó una ligera disminución porcentual entre los dos años de 8.8\% y un crecimiento promedio para 11 años de $2.4 \%$. De lo anterior se puede concluir que en el Distrito de Cartagena, la violencia homicida se ha mante- 
nido relativamente constante con una ligera tendencia al decrecimiento en los casos registrados desde el año 1995 hasta el año 2005.

GRÁFICA No. 1. EVOLUCIÓN ANUAL DE MUERTES POR HOMICIDIOS EN CARTAGENAY TASA DE HOMICIDIOS. 1995 - 2005

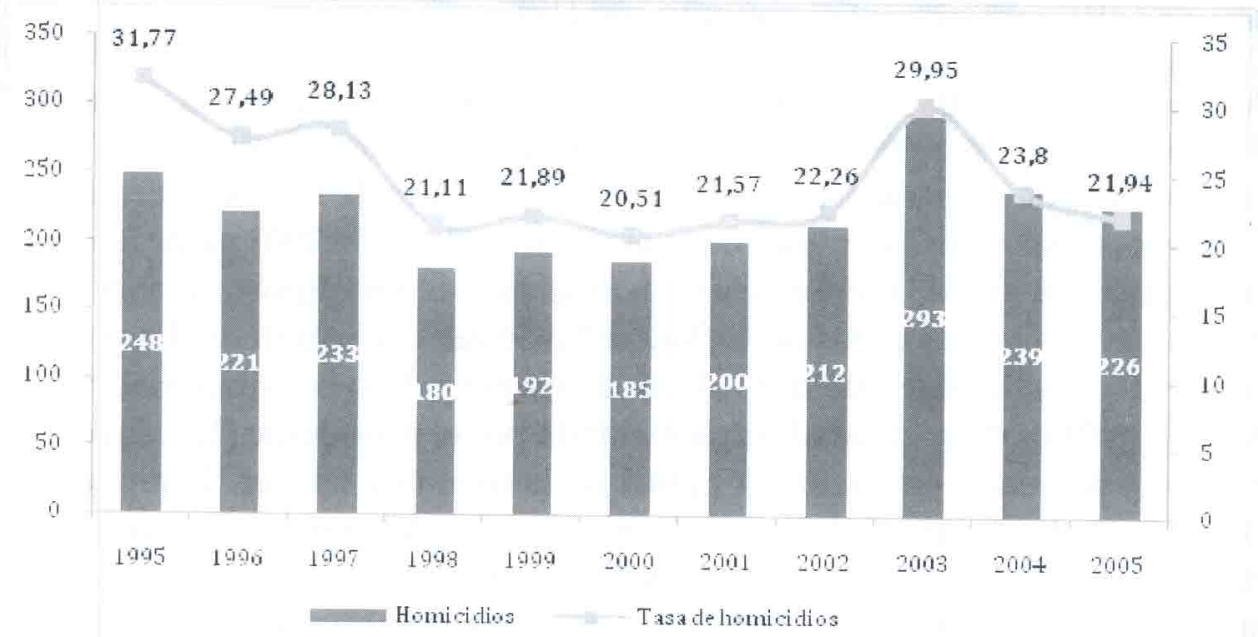

Fuente: Elaboración propia con base en COSED y Medicina Legal

La gráfica muestra además que durante la década de los 90, la incidencia de casos de homicidios presentaba una tendencia a la baja, alcanzando valores de 180 casos en 1998, cifra inferior en aproximadamente $22.7 \%$ con respecto al año inmediatamente anterior e inferior a su vez en $27.4 \%$ con respecto a la cifra de 1995. A partir del año 1999, el número de muertes por homicidios revierte su tendencia y empieza a incrementarse (aunque disminuye ligeramente en el 2000) y pasa de 192 a 293 casos en el 2003, lo que representó un aumento porcentual del $52.6 \%$ entre los dos años. El determinante más próximo de este comportamiento lo constituye la crisis económica registrada en 1999 que elevó los niveles de pobreza y desempleo en el país de manera considerable lo que pudo repercutir en aumento en las tasas delincuenciales, no sólo de Cartagena sino de algunas otras ciudades del país. Un artículo realizado por PROEXPORT Colombia (14), para este mismo periodo, muestra una comparación de las tasas de homicidios de Cartagena y las principales ciudades de América, tomando un promedio de tasas para estos años (1999 - 2003), donde se observa que Cartagena se ubica como la cuarta ciudad de mayor tasa de homicidios, 24 por cada 100 mil habitantes, precedida por Medellín, Washington y Brasilia, que registran respectivamente tasas de 104, 62 y 38 por cada 100 mil habitantes. 


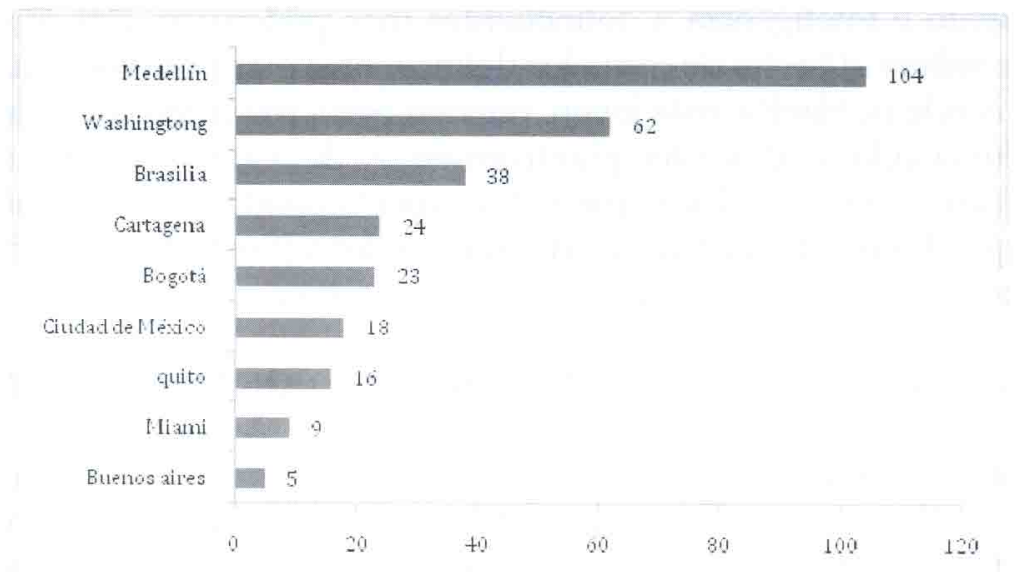

Fuente: PROEXPORT, Colombia. "La Inversión Extranjera y el Turismo hacia Colombia".

Lo más importante de realizar un análisis a la situación de la violencia homicida durante esta última década, es encontrar posibles relaciones de causalidad que ayuden a explicar el comportamiento de la tasa de homicidios. La Hipótesis sobre las causas objetivas de la violencia, desarrollada por la Comisión de Estudios Sobre La Violencia, es tal vez una de la más acertada al momento de explicar la situación Cartagenera. En ella se establece la idea de que las verdaderas causas de la violencia en el caso colombiano son la Pobreza, la falta de educación y la desigualdad del pueblo y utiliza como variables para explicar esta aseveración el Índice de Calidad de Vida (ICV), el coeficiente de Gini, promedio de escolaridad de la fuerza laboral y la tasa de participación electoral de los alcaldes municipales. En este sentido, podríamos decir que la incidencia de homicidios podría estar en gran parte explicada por la teoría de las causas objetivas puesto a que la mayor frecuencia de casos, durante los últimos 11 años, se encuentra distribuida en los barrios categorizados como "los más pobres" como Olaya Herrera, El Pozón y Nelson Mandela, que son considerados barrios de estratificación 1 , que a su vez son los barrios que registran los menores niveles de ICV y las tasas más bajas de escolaridad.

Otra posible explicación al fenómeno de la violencia homicida que vive Cartagena, podría encontrarse en el artículo de Gaviria (2000) (15), quien basado en las hipótesis de Becker reseña algunas causas que explican el comportamiento de las tasas de Homicidios, a partir de 3 modelos aislados. En el primero simplemente se argumenta la forma en que el delincuente puede, de forma potencial, influir en la sociedad y hacer que un residente particular quiera vincularse a la realización de actividades delictivas, lo 
que a su vez, disminuye la probabilidad de castigo de los mismos. En el segundo modelo se plasma la idea de cómo los delincuentes transmiten su conocimiento e inteligencia a delincuentes más poderosos. Por último el tercero se refiere al hecho de cómo los delincuentes más poderosos, al tener contacto con la población más joven, pueden pervertir la moral social de la zona influenciada. Todos estos planteamientos, de alguna manera, constituyen la base sobre la cual se explica el comportamiento que han tenido las muertes por homicidio en Cartagena durante los últimos 11 años, dada la estructura y situación social que se vive en el Distrito.

\section{PERFIL CARACTERÍSTICO DE LOS HOMICIDIOS EN CARTAGENA}

La gráfica No. 3 muestra la frecuencia de casos de homicidios por 4 rangos horarios. Se observa, en primer lugar, que en 759 de los casos no se obtuvo información acerca de la hora exacta de ocurrencia del hecho, mientras que 742 víctimas, representando el 30.5\%, fueron asesinadas en las horas de la noche, entre las 18:00 y las 23:59, constituyéndose en el rango horario de mayor ocurrencia de casos durante el periodo analizado.

De igual forma, 453 (18.6\%) de las víctimas fueron ultimadas en las horas de la tarde (entre las 12: y las 17:59); 179 (7.3\%) en las horas de la mañana y otras 296 (12.1\%) en las horas de la madrugada. En este sentido, sería pertinente la utilización de medidas que ayuden a controlar la delincuencia de manera mucho más intensiva en las horas nocturnas, operativos de seguridad en zonas específicas de alto riesgo, programas de prevención teniendo en cuenta el criterio y las consideraciones de la población afectada.

GRÁFICA No. 3. HOMICIDIOS EN CARTAGENA POR HORA DE OCURRENCIA 1995 - 2005

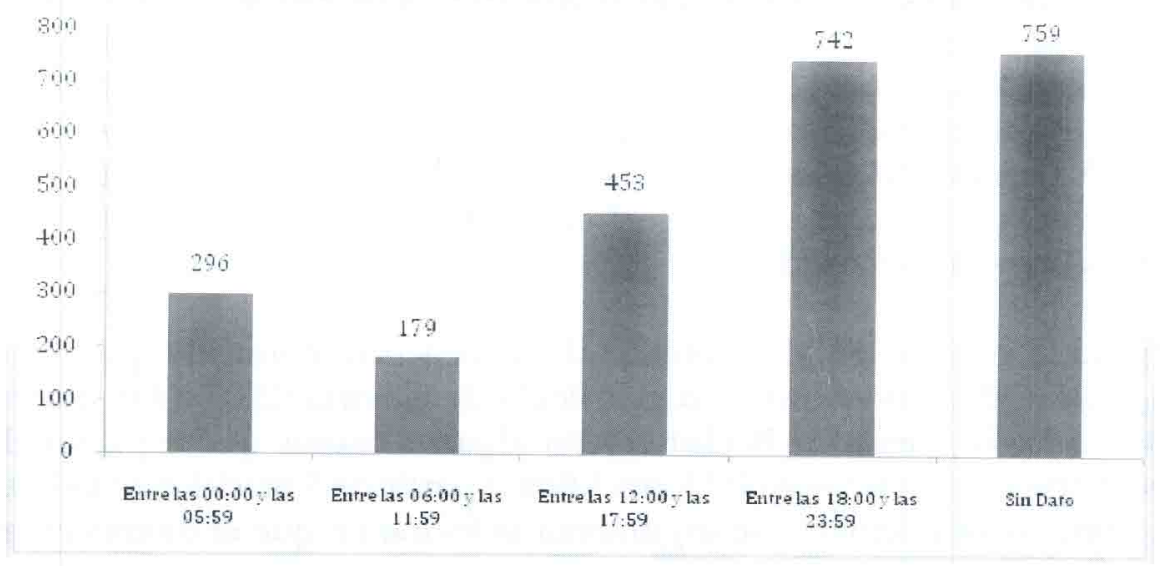

Fuente: Elaboración propia con Base en COSED 


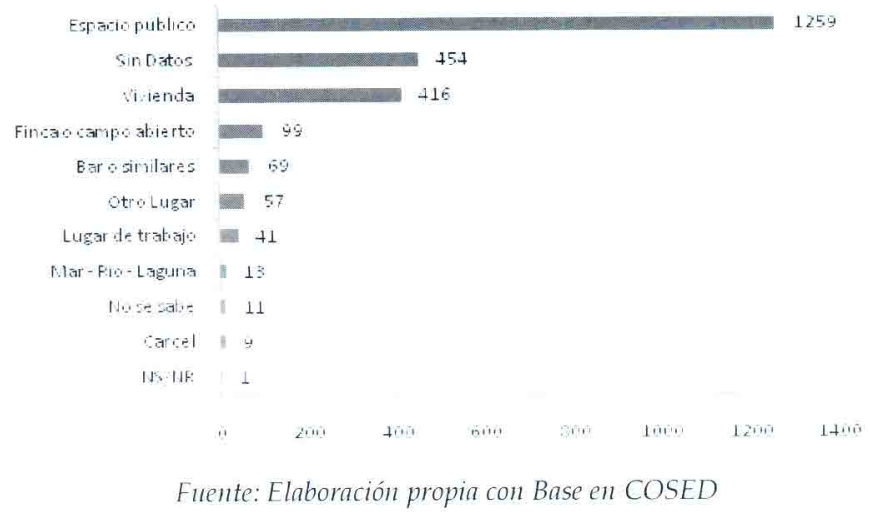

Así mismo, la gráfica No. 4 muestra la distribución de frecuencia del total de homicidios según lugar de ocurrencia. Se observa que en 1259 de las ocasiones, representando el (51.8\%), la víctima fue asesinada en el espacio público, poniendo de evidencia la peligrosidad que tienen las calles de algunas zonas de la ciudad.

De igual forma, la vivienda se constituye en el segundo lugar de mayor frecuencia de casos que se han registrado durante el periodo de análisis, reportando un total de 416 casos. Lo preocupante en este sentido es observar que en algunas partes de la ciudad la vivienda no representa un lugar seguro para algunas personas puesto que sencillamente la delincuencia se aferra en violar el derecho a la propiedad y a la vida. Por otro lado, cabe señalar que en 99 casos la víctima es asesinada en una finca o campo abierto (4\%), 69 en bares o similares, 13 en río o laguna, 9 en la cárcel, 41 en su lugar de trabajo, y en 454 de los casos no se obtuvo información al respecto.

GRÁFICA No. 5. HOMICIDIOS EN CARTAGENA POR CONTEXTO DEL HECHO 1995 - 2005

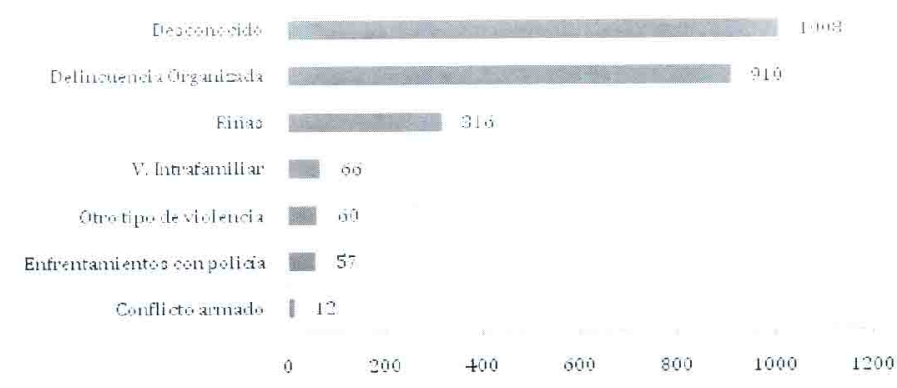

Fuente: Elaboración propia con Base en COSED 
Como delincuencia organizada, se categorizan todos aquellos casos de homicidios cometidos bajo los móviles de sicariato, secuestro, ajuste de cuentas, hurto simple, intolerancia, entre otros (5). La cifra para este contexto es de 910 , representando $37.4 \%$ del total y constituyéndose en el segundo de mayor frecuencia, teniendo en cuenta que en 1008 de los casos $(41.4 \%)$ no se obtuvo información sobre el contexto de los hechos o simplemente no se pudo establecer una versión clara de los mismos.

En otros 57 casos, la víctima es ultimada en enfrentamiento con organismos de seguridad oficial y en 66 es ultimada en violencia intrafamiliar. Las riñas constituyen el principal componente de la categoría violencia interpersonal, reportando un total de 316 casos; así mismo en conflicto armado murieron 12 personas y otras 54 fueron asesinadas mediante otro tipo de violencia como por ejemplo una bala perdida.

TABLA No. 1. HOMICIDIOS POR EDAD Y SEXO. CARTAGENA DE INDIAS 1995 - 2005

\begin{tabular}{|c|c|c|c|}
\hline RANGO & MASCULINO & FEMENINO & TOTAL \\
\hline $1-4$ & 8 & 1 & 9 \\
\hline $5-9$ & 4 & 3 & 7 \\
\hline $10-14$ & 20 & 2 & 22 \\
\hline $15-19$ & 260 & 21 & 281 \\
\hline $20-24$ & 470 & 25 & 495 \\
\hline $25-29$ & 399 & 20 & 419 \\
\hline $30-34$ & 320 & 19 & 339 \\
\hline $35-39$ & 285 & 10 & 295 \\
\hline $40-44$ & 185 & 15 & 200 \\
\hline $45-49$ & 117 & 6 & 123 \\
\hline $50-54$ & 61 & 4 & 65 \\
\hline $55-59$ & 28 & 4 & 32 \\
\hline $60 \cdot 64$ & 17 & 1 & 18 \\
\hline $65-69$ & 31 & 1 & 32 \\
\hline SIN DATO & 85 & 7 & 92 \\
\hline TOTALES & 2290 & 139 & 2429 \\
\hline
\end{tabular}


Se hace necesario notar, que durante 1995 - 2005 ha prevalecido el homicidio a personas del sexo masculino, los cuales concentran el $94.2 \%$ del total. Por otro lado, las muertes a personas de sexo femenino suman en total 139, $5.4 \%$, y en 6 de los incidentes no se pudo establecer el sexo de la víctima en cuestión. Además de ello, se puede notar en la Tabla No. 1 que el rango de edad con mayor número de víctimas lo constituye el de 20 a 29 años, reportando 914 casos. De igual forma, 634 víctimas tenían edades de entre 30 y 39 años al momento de ser ultimadas, 323 entre 40 y 49 años y 97 entre 50 y 59 años, conformando los rangos etáreos de mayor frecuencia.

La conclusión más relevante y fácilmente observable, a partir del análisis de las dos anteriores variables, es la que sin duda representa una problemática social en tanto que la población más afectada por el fenómeno de los homicidios se encuentra representada en su gran mayoría por jóvenes de sexo masculino con edad de entre 20 y 30 años.

A su vez es importante resaltar la cifra obtenida a partir de las víctimas que tenían edades de entre 10 y 19, ya que estas muertes representan cerca del $12.4 \%$ del total. En este sentido es preocupante observar que estos dos rangos etáreos concentran el periodo de "alto desarrollo y productividad de la vida de los individuos lo cual repercute en un obvio impacto económico debido a las aportaciones de las que se priva la sociedad" (6).

A continuación, se tratará de analizar una de las variables que más caracteriza a la violencia homicida en Cartagena, y que tal vez sea una de las más relevantes para los actores de las políticas públicas locales: el barrio de ocurrencia de los hechos. Cartagena de indias está conformada por 3 localidades, las cuales concentran a su vez, 15 comunas urbanas y 15 comunas rurales, las cuales se desagregan en cerca de 180 barrios. La gráfica No. 6 muestra la distribución de frecuencias de casos de homicidios para los 11 barrios de mayor ocurrencia.

Adicionalmente, Se muestra en la gráfica que los barrios Olaya Herrera y el Pozón, con 169 y 154 casos respectivamente, concentran el 13.2\% del total de casos por barrio de ocurrencia. Seguido a estos, en orden de frecuencia, Torices (78 casos), La Esperanza (56 casos), La Candelaria (55 casos) y Barrio Chino (48 casos), todos catalogados como barrios en promedio 1, 2 y 3 de estratificación social según el informe de la Secretaría de Planeación Distrital.

Lo anterior se hace evidente a partir de este análisis es el hecho de que pueda existir una relación alta y de causalidad entre los niveles de pobreza y la violencia, puesto que a medida que aumenta la estratificación socioeconó- 
mica, disminuyen de forma significativa los casos de homicidios y viceversa. Algo de ello fue tratado por James Wilson y George Kelling en 1982 (12), en la denominada teoría de Broken Windows. Esta teoría que ha sido probada más de una vez sostiene que los espacios públicos con mayor nivel de deterioro y desorden conducen a mayores niveles de crimen y violencia.

Teniendo en cuenta este planteamiento, se encuentra que Olaya Herrera y El Pozón presentan los niveles más deplorables en las condiciones de vida de sus habitantes y el desarrollo de su infraestructura tanto comercial como patrimonial coincide en gran parte con lo que se plantea en la Broken Windows.

Los barrios con mayor desarrollo cultural, económico, social y patrimonial son los que se ubican en la localidad Histórica y del Caribe Norte, barrios como Bocagrande, El Laguito, Manga, entre otros, que en los 11 años de estudios resultan ser los de menor frecuencia de casos, sumando entre si un total de 32 casos, según los reportes del COSED.

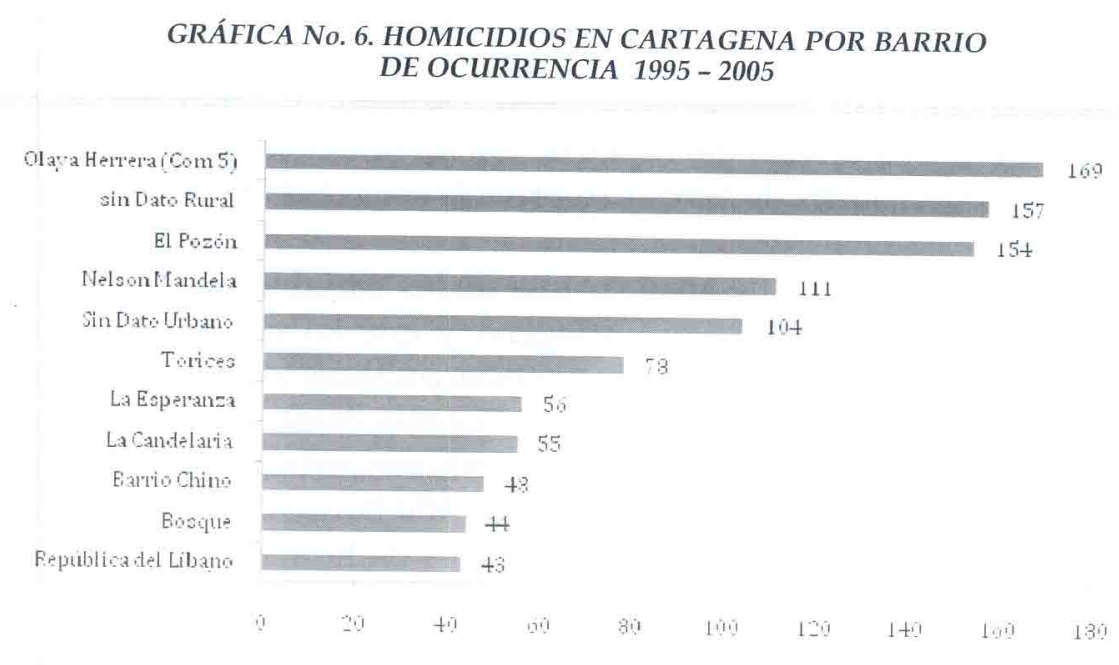

Fuente: Elaboración propia con Base en COSED

Dadas estas características que le dan un toque de particularidad y singularidad al caso cartagenero, sería importante considerar la orientación de los recursos públicos en materia de seguridad, prevención, reordenamiento territorial y asistencia pública hacia estas zonas marginadas que presentan una gran incidencia de violencia, criminalidad y desplazamiento, lo cual podría repercutir en una mejora significativa de estos índices que hoy afectan gran parte del atractivo turístico de la ciudad. 


\section{DETERMINACIÓN DE LOS COSTOS ECONÓMICOS DE LO HOMI- CIDIOS: AÑNOS POTENCIALES DE VIDA PERDIDOS}

Una vez procesada la información bajo el software EPIDAT 3.1, utilizando datos poblacionales por grupos etáreos quinquenales y el número de defunciones para cada uno de éstos rango etáreos correspondientes a la ciudad de Cartagena de Indias se obtuvieron los siguientes resultados:

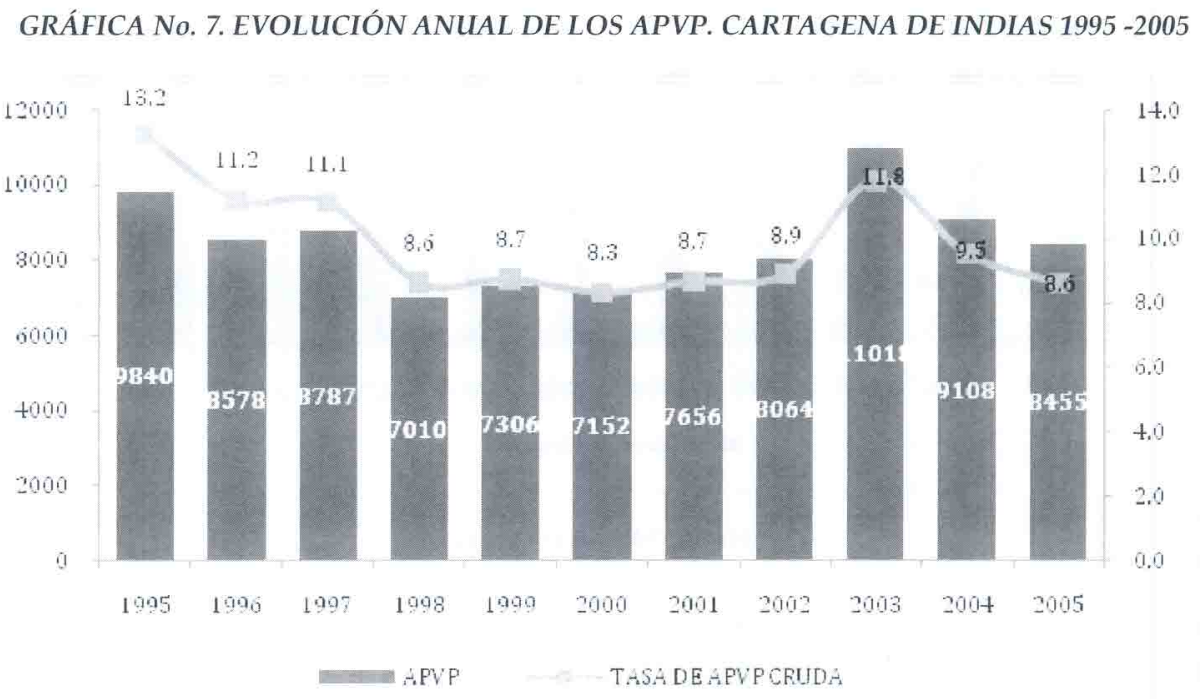

Fuente: Elaboración propia

Entre los años 1995 y 2005 se obtuvo un promedio anual de 8.452 APVP, siendo 11.018 el valor máximo alcanzado en el año 2003 y 7.010 el valor mínimo el cual se obtuvo en el año 1998. La interpretación correcta de este indicador para este caso sería: para el año 2005, por ejemplo, de las 226 muertes por homicidios ocurridas se obtuvo un total de 8.455 Años Potenciales de Vida Perdidos.

La gráfica No. 9 muestra, con una línea de color azul y medida en el eje vertical de la derecha, la Tasa de APVP cruda la cual representa el promedio de años perdidos por cada 1000 muertes por homicidio. El valor de esta osciló entre 13.21 (valor máximo alcanzado) en 1995 a 8.6 en el 2005, con una media anual 9.87 y un valor mínimo de 8.3 alcanzado en el año 2000. Hay que hacer notar que a pesar de que el año 2003 reporta el mayor número de APVP, la mayor tasa se registró en el año 1995 lo cual se explica por la razón 
de que en dicho año las víctimas de menor edad fueron proporcionalmente mayores que las del año 2003, es decir, hubo más víctimas jóvenes en 1995 que en el 2003.

GRÁFICA No. 8. DISTRIBUCIÓN DE APVP POR GÉNERO. 1995 - 2005

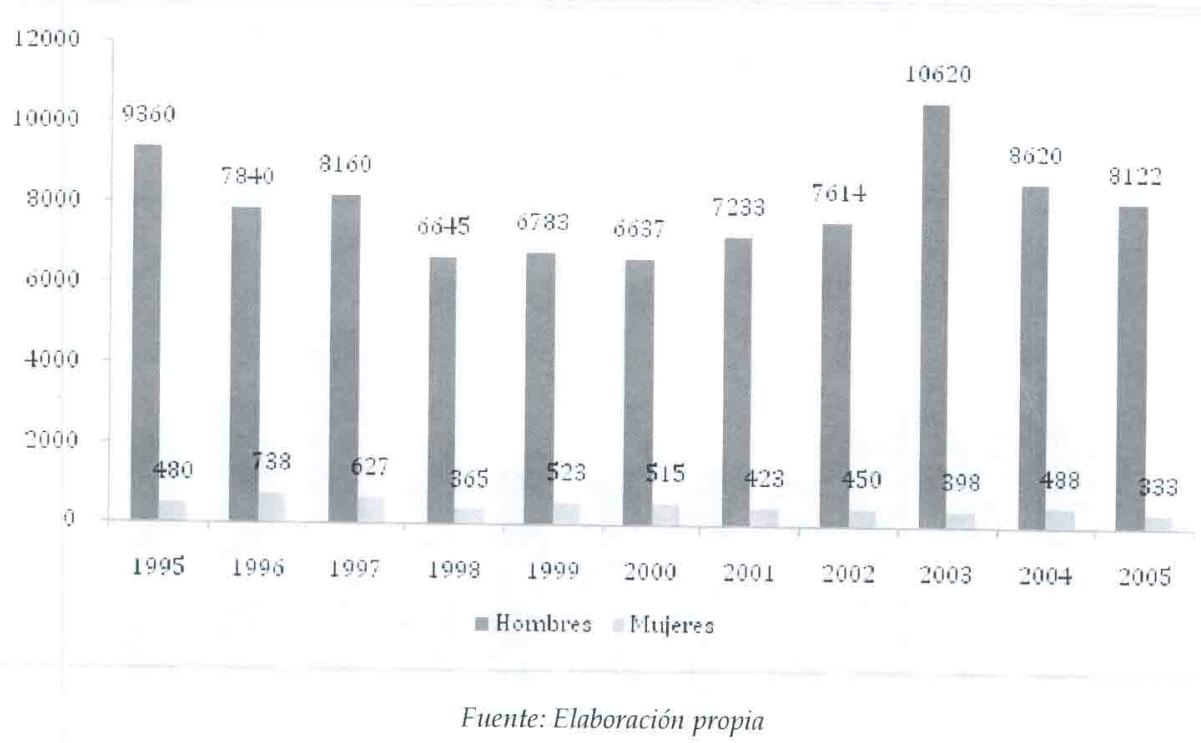

Así mismo los resultados nos permiten observar la distribución de los Años Potenciales de Vida Perdidos según el género. A partir de la gráfica No. 8 se puede observar que los APVP correspondientes al caso de los hombres concentran ampliamente más del 90\% del total de APVP para todos los años, con un valor promedio de 7.967 y con valores máximo y mínimo de 10.620 y 6.637 obtenidos en los años 2003 y 2000 de manera respectiva. Para el caso de las mujeres por el contrario, se obtuvo un promedio anual de 485 APVP con valores máximo y mínimo de 738 y 333 respectivamente.

En el sentido estrictamente económico, esta distribución desproporcionada de APVP por géneros podría incidir en la productividad ya que la oferta laboral disponible tiene como actores principales a los hombres si consideramos que tradicionalmente las mujeres han sido las encargadas de las labores del hogar, eso sí, sin menospreciar la creciente participación que se ha presentado en los últimos años de la mujer en los mercados laborales ${ }^{4}$.

\footnotetext{
${ }^{4}$ Las cifras del DANE muestran que para el periodo Sept - 2006, el 57.9\% de las fuerza laboral disponible para Cartagena estaba constituida por hombres y solo el $42.1 \%$ era representado por las mujeres
} 
La gráfica No. 9, muestra la evolución de la Tasa de APVP cruda por géneros midiendo en el eje vertical de la derecha la correspondiente al caso de las mujeres y en el eje de la izquierda la de los hombres. Lo que se observa en primera instancia es la alta diferencia entre tasas: la de los hombres osciló entre los 26.31 en 1995 y 17.29 en el 2005 con un valor promedio de 19.46 y en cambio la de las mujeres escasamente alcanza 1.8 como valor máximo en 1996. La interpretación de estas cifras nos muestra que en el 1995 por cada 1.000 víctimas de sexo masculino se perdieron en promedio 26.31 APVP siendo ésta la mayor cifra alcanzada en todo el periodo analizado. Las mujeres por el contrario reportan la mayor tasa de APVP en 1996 la cual indica que por cada 1.000 víctimas de sexo femenino se perdió en promedio 1.84 años.

La razón de esta diferencia de tasas puede no solo estar en el número de casos reportados según el sexo en todos y cada uno de los años del periodo analizado, sino que una posible explicación podría hallarse en lo que llamaremos las "diferencias de causas". La diferencia de causas puede existir, por ejemplo, si generalmente asumimos que el rol que desempeña el hombre dentro de su contexto económico - social y su responsabilidad para con la sociedad, aumenta la probabilidad de ser asesinado por razones que obviamente tienen que diferir en el caso de las mujeres.

GRÁFICA No. 9. TASA DE APVP CRUDA POR GÉNEROS. CARTAGENA DE INDIAS 1995 -2005

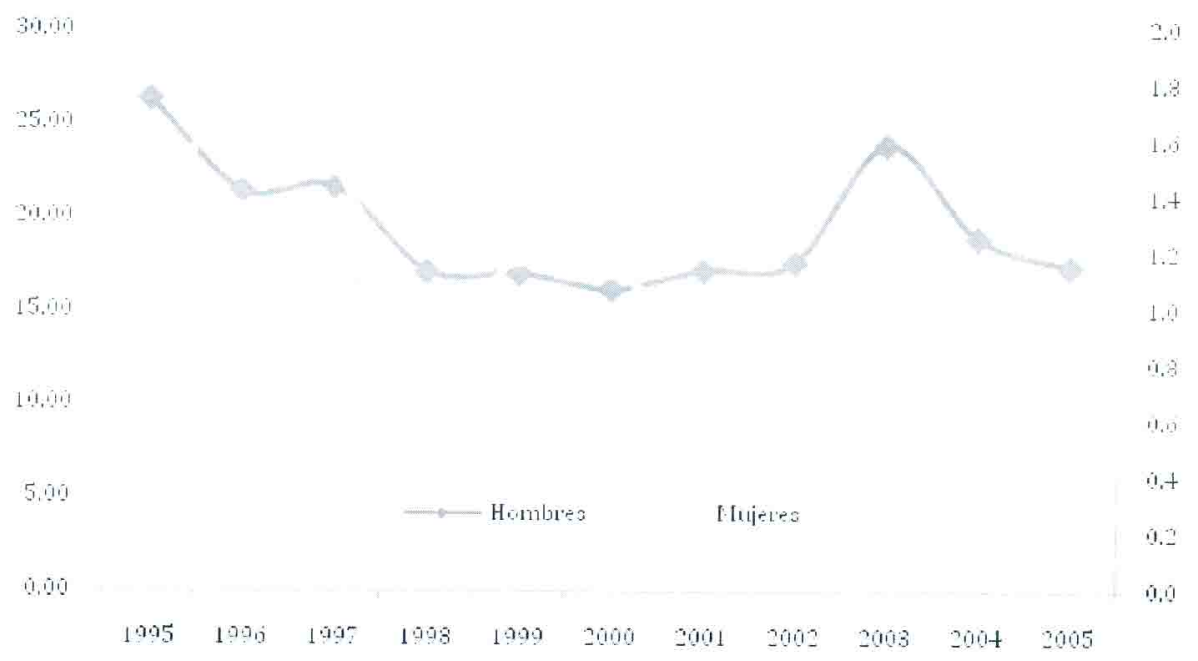

Fuente: Elaboración propia 
Ahora bien, si nuestro fin último es aproximarnos a una estimación que recoja el valor monetario de los Años Potenciales de Vida Perdidos para obtener el costo que para la sociedad representa una muerte por homicidio, como es nuestro propósito, podríamos tomar como PROXY de los ingresos no percibidos durante los años que se esperaban vivir el PIB per cápita nacional ${ }^{5}$ y multiplicarlos directamente por la cantidad de años obtenidos en el indicador. Los resultados obtenidos para el caso de Cartagena de Indias para el periodo en cuestión fueron los siguientes.

La tabla No. 2 muestra, que durante el periodo 1995 - 2005 el costo económico de los homicidios en Cartagena se estimaba en un promedio de 34.752 millones de pesos colombianos con valores máximo y mínimo de \$56.474 millones y 21.558 millones alcanzados en los años 2003 y 1998 respectivamente. Hay que tener en cuenta que al utilizar el valor del PIB per cápita estamos asumiendo que todas las personas asesinadas percibían por lo menos esta cantidad de ingresos anuales y por tanto la interpretación de las cifras estimadas como costos económicos deberían ser consideradas como "mínimas".

Otra consideración de importancia, en el análisis de los resultados es la de tener en cuenta la no variabilidad de los ingresos a lo largo de los años esperados de vida y asumir que estos permanecen constantes a lo largo del tiempo, supuesto que parece alejarse un poco de la realidad observada en cuanto sabemos que a medida que las personas incrementan su productividad y su capital humano las variaciones en el ingreso se comportarán de manera proporcional. Sin embargo hay que decir que el análisis aquí presentado hace referencia a un modelo específico con todas sus limitaciones propias de cualquier modelo.

Por otro lado, si nuestro interés reside en encontrar el valor económico de los Años Potenciales de Vida Perdidos para todo el periodo analizado debemos llevar a precios de un solo año toda la serie de flujos anuales. Para este caso tomaremos todos y cada uno de los valores obtenidos como costos y calcularemos su valor en el año 2005 utilizando los datos disponibles de la inflación y la fórmula de valor presente en cada uno de ellos. Los cálculos realizados arrojaron los resultados que se muestran en la tabla No. 3.

\footnotetext{
${ }^{5}$ Con exacto rigor, las cifras correspondientes al PIB per cápita local no han sido estimadas de manera cabal. Por eso en el presente estudio se utiliza el PIB per cápita nacional que de igual forma nos muestra la cantidad de ingresos que percibe cada persona dentro del territorio nacional.
} 
TABLA No. 2. DETERMINACIÓN DE LOS COSTOS ECONÓMICOS DE LOS HOMICIDIOS. CARTAGENA DE INDIAS. 1995 - 2005

\begin{tabular}{cccccc} 
& & \multicolumn{2}{c}{ PIB per cápita Nacional } & \multicolumn{2}{c}{ Costos Económicos } \\
AÑOS & APVP & Pesos & Dólares & Pesos & Dólares \\
1995 & 9840 & $\$ 2.190 .855$ & $\$ 2.400$ & $\$ 21.558$ & $\$ 23,62$ \\
1996 & 8578 & $\$ 2.562 .905$ & $\$ 2.473$ & $\$ 21.985$ & $\$ 21,21$ \\
1997 & 8787 & $\$ 3.037 .820$ & $\$ 2.662$ & $\$ 26.693$ & $\$ 23,39$ \\
1998 & 7010 & $\$ 3.440 .957$ & $\$ 2.411$ & $\$ 24.121$ & $\$ 16,90$ \\
1999 & 7306 & $\$ 3.644 .352$ & $\$ 2.072$ & $\$ 26.626$ & $\$ 15,14$ \\
2000 & 7152 & $\$ 4.132 .574$ & $\$ 1.980$ & $\$ 29.556$ & $\$ 14,16$ \\
2001 & 7656 & $\$ 4.377 .890$ & $\$ 1.904$ & $\$ 33.517$ & $\$ 14,57$ \\
2002 & 8064 & $\$ 4.641 .394$ & $\$ 1.851$ & $\$ 37.428$ & $\$ 14,92$ \\
2003 & 11018 & $\$ 5.125 .578$ & $\$ 1.781$ & $\$ 56.474$ & $\$ 19,63$ \\
2004 & 9108 & $\$ 5.686 .594$ & $\$ 2.165$ & $\$ 51.794$ & $\$ 19.72$ \\
2005 & 8455 & $\$ 6.211 .913$ & $\$ 2.677$ & $\$ 52.522$ & $\$ 22,63$
\end{tabular}

Fuente: Elaboración propia con base en COSED y BANREP

La nueva serie registra un promedio anual de 48.253 millones de pesos colombianos a precios del año 2005, al igual que un total de $\$ 530.778$ millones y valores máximo y mínimo de $\$ 62.469$ millones en el 2003 y \$38.874 millones en 1998. TABLA No. 3. OBTENCIÓN DEL VALOR TOTAL DE LA SERIE ANUAI
DE COSTOS. CARTAGENA DE INDIAS. $1995-2005$

\begin{tabular}{cccc} 
Año & Pesos Corrientes & \multicolumn{2}{c}{ Costos Económicos } \\
1995 & $\$ 21.558$ & Inf & Pesos (2005) \\
1996 & $\$ 21.985$ & 19,46 & $\$ 58.034$ \\
1997 & $\$ 26.693$ & 21,63 & $\$ 48.658$ \\
1998 & $\$ 24.121$ & 17,68 & $\$ 50.203$ \\
1999 & $\$ 26.626$ & 16.7 & $\$ 38.874$ \\
2000 & $\$ 29.556$ & 9,23 & $\$ 39.284$ \\
2001 & $\$ 33.517$ & 8,75 & $\$ 40.099$ \\
2002 & $\$ 37.428$ & 7,65 & $\$ 42.242$ \\
2003 & $\$ 56.474$ & 6,99 & $\$ 44.089$ \\
2004 & $\$ 51.794$ & 6,49 & $\$ 62.469$ \\
2005 & $\$ 52.522$ & 5,5 & $\$ 54.305$ \\
& TOTAL & 4,85 & $\$ 52.522$ \\
& & & $\$ 530.778$
\end{tabular}

Fuente: Elaboración propia con Base en DANE 
Desde el punto de vista de la racionalidad económica, existe una diferencia desproporcionada en la relación Costo Beneficio, si consideramos el hecho de que actualmente el Distrito de Cartagena dispone de ingresos de aproximadamente 9.000 millones de pesos al año para atender su gasto en seguridad ${ }^{6}$ evidenciando una pérdida significativa si tenemos en cuenta que la sociedad reporta costos promedios anuales, por motivos de muertes por homicidios solamente, de 48.253 millones de pesos. Este análisis nos permite plantear un par de hipótesis algo que serán profundizadas en trabajos posterioes: 1) Nos encontramos en una situación en la que los ingresos son insuficientes y por tanto la productividad de las empresas encargadas de prestar el servicio de seguridad a los ciudadanos sea de igual forma muy baja debido a los altos costos que le reporta a la sociedad el delito y la violencia; o 2) La eficiencia en el uso de los recursos disponibles no alcanza su óptimo por diversas razones de entorno provocando una desmejora en la prestación del servicio de seguridad lo que repercute negativamente sobre los índices de violencia y por tanto sobre los costos sociales que de ella se derivan.

Por último, hay que mencionar que el análisis de los APVP nos permite además realizar comparaciones de tasas obtenidas para este indicador siempre y cuando se realice un ajuste en las tasas de APVP crudas obtenidas anteriormente. Para ello recurrimos al método directo de estandarización utilizado en diversos estudios epidemiológicos y de salud y con el cual se pretende calcular "la tasa que se esperaría encontrar en las poblaciones bajo estudio si todas tuvieran la misma composición según la variable cuyo efecto se espera ajustar o controlar" (9).

Para ello se utiliza una población estándar o de referencia de la cual dependerá en gran parte el valor que se obtenga para la tasa de APVP ajustada, por lo tanto la escogencia de la población estándar debe hacerse teniendo en cuenta que ésta utilice más o menos la misma proporcionalidad por grupos etáreos que nuestra población objetivo. Por otro lado, también habría que mencionar el hecho de que estas poblaciones no son calculadas utilizando valores observados, por tanto no medirán una magnitud absoluta sino simplemente para la comparación entre grupos de diferentes poblaciones. Al ajustar la tasa de APVP cruda obtenida utilizando la "población mundial estándar de Waterhouse" 7 .

\footnotetext{
${ }^{6}$ De estos ingresos dependen tanto la formulación de políticas públicas integrales que beneficien a la sociedad en general, como la orientación de los recursos a aquellas zonas que presenten los más altos índices de criminalidad y violencia incluidos los homicidios que, sin lugar a dudas, constituye uno de los principales ejes de atención en el análisis del delito en todas sus dimensiones.

${ }^{7}$ Esta población ha sido utilizada por la OMS y la OPS en diversos estudios. Es utilizada aquí por tener una proporcionalidad de jóvenes muy similar al caso cartagenero
} 


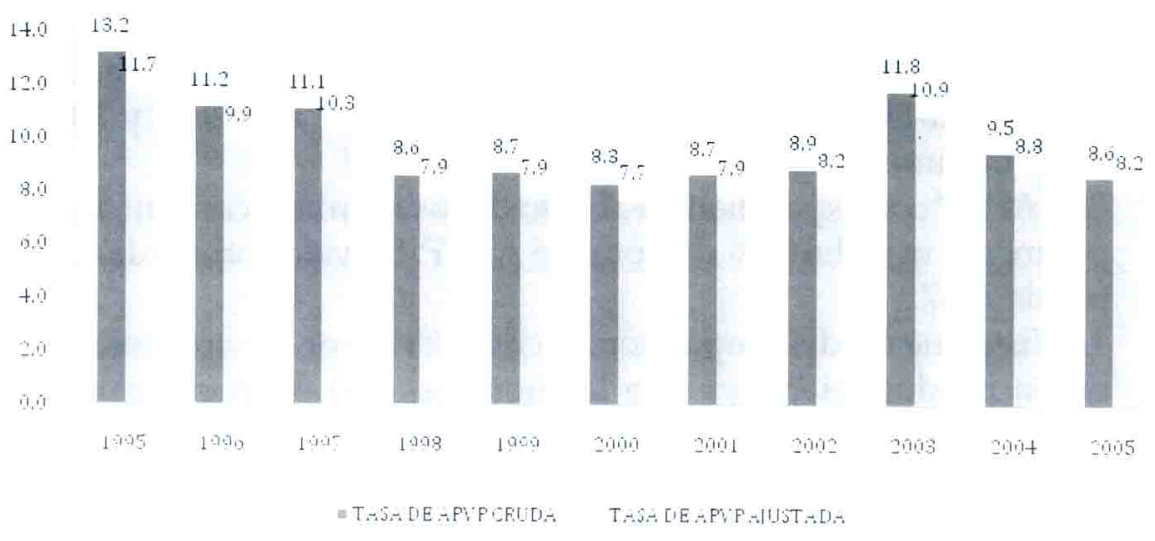

Filente: Elaboración propia con base en Waterhouse J. y Col. (Eds.) Cancer incidence in froe continents. I yon, IARC, 1976. OMS y OPS

La gráfica No. 10 muestra, que para el año 2005, por ejemplo, si la población cartagenera presentara la misma estructura que la población de referencia se obtendrían en promedio por cada 1000 muertes por homicidios un total de 8.2 APVP. En promedio anual, esta tasa alcanza valores de 9.02 APVP, valores máximos y mínimo de 11.66 y 7.68 respectivamente con una desviación estándar de 1.41.

\section{CONCLUSIONES}

$\checkmark \quad$ En Cartagena de Indias durante 1995 - 2005, ocurrieron un total de 2429 muertes por homicidios, de las cuales 226 ocurrieron durante el último año y 248 casos en el primero.

$\checkmark \quad$ El mes de diciembre ha sido el de mayor ocurrencia de casos de homicidios, reportando 249 casos de los 2429 y representando el $10.25 \%$ del total.

$\checkmark \quad$ El rango horario de mayor ocurrencia de casos durante el periodo analizado fueron las horas de la noche, entre las 18:00 y las 23:59, el cual reportó 742 víctimas (30.5\%)

$\checkmark \quad$ El 51.8\% de las víctima fueron asesinadas en el espacio público. Por otro lado la vivienda se constituye en el segundo lugar de mayor frecuencia de casos con un total de 416.

$\checkmark$ Durante 1995 - 2005 ha prevalecido el homicidio a personas del sexo masculino, los cuales concentran el $94.2 \%$ del total. Por otro lado, las muertes a personas de sexo femenino suman en total 139, $5.4 \%$

$\checkmark$ Para el caso de Cartagena, es probable que pueda existir una relación 
alta y de causalidad entre los niveles de pobreza y la violencia, puesto que a medida que aumenta la estratificación socioeconómica, disminuyen de forma significativa los casos de homicidios y viceversa.

$\checkmark$ Entre 1995 y el 2005 se obtuvo un promedio anual de 8.452 APVP, siendo 11.018 el valor máximo alcanzado en el año 2003 y 7010 el valor mínimo el cual se obtuvo en el año 1998.

$\checkmark$ Los APVP correspondientes al caso de los hombres concentran ampliamente más del 90\% del total de APVP. Su valor promedio anual fue de 7967.

$\checkmark$ La distribución desproporcionada de APVP por géneros incide sobre la productividad ya que los hombres son proporcionalmente mayores dentro la oferta laboral disponible.

$\checkmark \quad$ Utilizando el PIB per cápita como PROXY de los ingresos no percibidos, durante el periodo 1995 - 2005 el costo económico de los homicidios en Cartagena se estimó en un promedio de 34.752 millones de pesos colombianos.

$\checkmark \quad$ El Valor total de toda la serie de costos económicos a precios del 2005 alcanzó una cifra igual a \$530.778 millones de pesos.

\section{REFERENCIAS BIBLIOGRÁFICAS}

1. Alvis N, De la Hoz F. Tendencias de la Mortalidad por enfermedades Infecciosas en Cartagena de Indias, Colombia, 1995 - 2000. Revista Salud Pública. 2004; 6(3): 235 - 252.

2. Gómez C. Economía y violencia en Colombia. En: Martínez A. Economía, Crimen y Conflicto. BSCH, Universidad de Alcalá y Universidad Nacional. 2001.

3. Becker G. Crime and Punishment: An Economic Approach. The Journal of Political Economy. 1968; 76.

4. Farah A. Fundamentos económicos de la criminalidad en Cartagena de Indias [Tesis de pregrado]. Cartagena de Indias. Universidad Tecnológica de Bolívar; 1997.

5. Goyeneche F, Mercado D. Aproximación a la caracterización de los homicidios en Cartagena. Investigaciones Centro de Observación y Seguimiento del Delito - COSED. 2006.

6. Centro de Observación y Seguimiento del Delito - COSED. Informe anual de Muertes por Causa Externa 2005. COSED. 2006.

7. Olavarría M, Contreras E. Costos económicos del delito en Chile. Ministerio del Interior, División de Seguridad Ciudadana [Web] 2005 [Consultado el 21 de septiembre de 2006]. Disponible en URL: http:/ / cronopio. flacso.cl/fondo/pub/digitalfree/2005/ libro/029509.pdf.

8. Silva I. Costos económicos de los delitos, niveles de vigilancia y políti- 
cas de seguridad ciudadana en las comunas del Gran Santiago. Instituto Latinoamericano y del Caribe de Planificación Económica y Social - ILPES [Web] 2000 [Consultado Junio de 2006]. Disponible en URL: http:/ / www. eclac.org/publicaciones/xml/9/4449/L-1328e.pdf.

9. Organización Panamericana de la Salud - OPS. La Estandarización: Un Método Epidemiológico Clásico para la Comparación de Tasas. Boletín Epidemiológico. 2002; 23 (3): 9-12.

10. Policía de Carreteras. Base de datos de reportes de movilidad [CDROM]. Cartagena de Indias. 2006

11. Departamento Nacional de Planeación - DNP. Reportes de evaluación. Sistema Nacional de Evaluación de Resultados. 2004; (13).

12. Wilson J, Kelling G. Broken Windows: the Police and Neighborhood safety. Atlantic Monthly. 1982; 249 (3), 29-38

13. Moreno A. Impacto del Transmilenio en el crimen de la Avenida Caracas y sus vecindades. Documento CEDE. Universidad de Los Andes. 2005; 55.

14. Proexport. La Inversión Extranjera y el Turismo hacia Colombia [Página Web]. Bogotá, Colombia: Proexport. 2005. [Acceso diciembre de 2006]. Disponible en: http://www.proexport.com.co/vbecontent/NewsDetail. asp?ID=7085\&IDCompany $=16$.

15. Gaviria A. Rendimientos crecientes y la evolución del crimen violento: el caso colombiano. En: Martínez A. Economía, Crimen y Conflicto. BSCH, Universidad de Alcalá y Universidad Nacional. 2001 
